\title{
465170 - INSPIRATORY SUPPORT IMPROVES PREOXYGENATION IN HEALTHY SUBJECTS
}

\author{
Issam Tanoubi, M.D., Pierre Drolet, MD, FRCPC, François Donati, PhD, MD, \\ FRCPC, Louis-Philippe Fortier, MSc, MD, FRCPC \\ Anesthesiology, Maisonneuve Rosemont Hospital , University Of Montreal, \\ Montreal, QC, Canada
}

Introduction: Preoxygenation optimizes oxygen content in the functional residual capacity (FRC). Creating a better reservoir with the help of inspiratory support and PEEP could improve gas exchange and shorten the time needed for adequate preoxygenation, defined as expiratory oxygen fraction (FEO2) greater than $90 \%$. The goal of this study was to compare the efficacy and tolerability of different levels of inspiratory support and PEEP during preoxygenation.

Methods: Local REB approval was obtained for this study. We conducted a double blind prospective randomized trial on 20 healthy volunteers aged between 20 to 40 years.

Subjects with body mass index $>30$, a mustache, a beard or claustrophobia were excluded. Under standard noninvasive monitoring we measured: inspiratory and expiratory oxygen fractions, end-tidal CO2, tidal and minute volumes, and inspiratory and endexpiratory pressures. Each subject went through three modes of preoxygenation in random order: normal breathing (SP), breathing under inspiratory support $4 \mathrm{cmH2O} / \mathrm{PEEP} 4 \mathrm{cmH} 2 \mathrm{O}$ (PSM-4) and inspiratory support 6 cmH2O /PEEP 4 cmH2O (PSM-6). Each mode lasted for 3 minutes and parameters were recorded every 10 seconds. We evaluated subject's tolerance, leaks and easiness of each mode of preoxygenation according to a simplified (1 to 4) scale. Two-way ANOVA was used to compare FEO2 while simple ANOVA was used for time to reach FEO2=90\%.

Results: More time was needed to reach FE02=90\% in group SP (144 $\pm 37 \mathrm{sec}$ ) compared to groups PSM-4 (95 \pm 34 sec, $\mathrm{P}<0.001)$ and PSM-6 (100 \pm 40 sec, $\mathrm{P}<0.001)$ (fig). Tidal volumes were higher in groups PSM-4 and PSM-6 $(\mathrm{P}<0.001)$. Spontaneous breathing and PSM-4 were better tolerated than PSM-6. Thirty-five\% of the SP group participants never reached an FE02=90\% compared to PSM-4: 0\% and PSM-6: 10\% (P=0.0061).

Discussion: Our study shows that combining inspiratory support and PEEP allows for a more efficient preoxygenation and faster FEO2 increase in healthy subjects. This may be attributed to larger tidal and minute volumes (1-2). This results in higher oxygen content in the FRC. Failure to reach FEO2 $=90 \%$ is usually the sign of incomplete denitrogenation of the FRC which happens in 25\% of healthy subjects during regular preoxygenation(3). It occurred less often in the PSM modes .Shortening the time needed to correctly preoxygenate patients may be of critical importance during emergencies, unsuspected difficult intubations and situations where positive pressure ventilation by mask is contraindicated. Inspiratory support 4cm during preoxygenation offers the same advantage than PSM-6 but is better tolerated.

References: 1-Anesth Analg 2004;98:1491-5 2-Anesthesiology 2005;102:1110-5 3British Journal of Anaesthesia 1994;72:116-118 


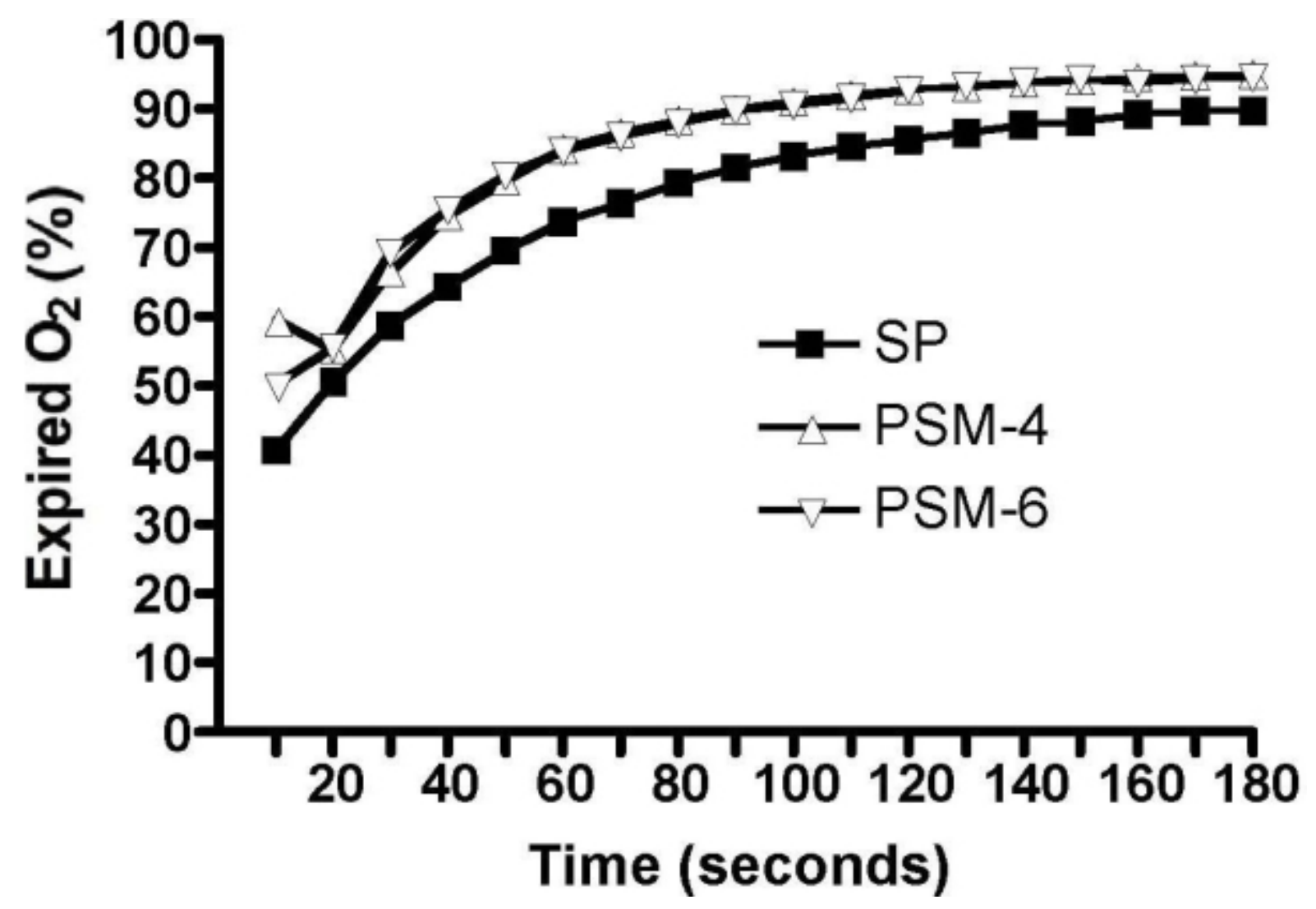

Expired O2 concentration. SP: spontaneous breathing; PSM-4: pressure support mode 4 cmH2O; PSM-6: pressure support mode 6 cmH2O. 